\title{
Detecting Myocardial Infarction by Electrocardiogram Machine Learning Models With Greater Accuracy; A Technical Advance Article
}

\section{M.D.S. Sudaraka}

Department of Obstetrics and Gynaecology, Faculty of Medicine, University of Peradeniya

\section{Abeyagunawardena}

Department of Physiology, Faculty of Medicine, University of Peradeniya

\section{E. S. De Silva}

Faculty of Medicine, University of Peradeniya

S Abeyagunawardena ( $\nabla$ shamali.agwd@yahoo.com )

Department of Medicine, Faculty of Medicine, University of Peradeniya

\section{Research Article}

Keywords: Myocardial infarction, Electrocardiogram, Machine learning models for Myocardial infarction detection.

Posted Date: January 29th, 2021

DOl: https://doi.org/10.21203/rs.3.rs-150700/v1

License: (9) This work is licensed under a Creative Commons Attribution 4.0 International License. Read Full License 


\section{Abstract}

\section{Background}

Electrocardiogram (ECG) is a key diagnostic test in cardiac investigation. Interpretation of ECG is based on the understanding of normal electrical patterns produced by the heart and alterations of those patterns in specific disease conditions. With machine learning techniques, it is possible to interpret ECGs with increased accuracy. However, there is a lacuna in machine learning models to detect myocardial infarction (MI) coupled with the affected territories of the heart.

\section{Methods}

The dataset was obtained from the University of California, Irvine, Machine Learning Repository. It was filtered to obtain observations categorized as Normal, Ischemic changes, Old Anterior MI and Old Inferior MI. The dataset was randomly split into a training set (70\%) and a test set (30\%). 73 out of the 270 ECG features were selected based on the changes observed following $\mathrm{MI}$, after excluding predictors that had near zero variance across the observations. Three machine learning classification models (Bootstrap Aggregation Decision Trees, Random Forest, Multi-layer Perceptron) were trained using the training dataset, optimizing for the Kappa statistic and the parameter tuning was achieved with repeated 10-fold cross validation. Accuracy and Kappa of the samples were used to evaluate performance between the models.

\section{Results}

The Random Forest model identified old anterior and old inferior Mls with 100\% sensitivity and specificity and all 4 categorized observations with an overall accuracy of 0.9167 (95\% Cl 0.8424 - 0.9633). Both the Bootstrap Aggregation Decision Trees and the Multi-layer Perceptron models identified old anterior Mls with $100 \%$ sensitivity and specificity and their overall accuracies for all 4 observations were $0.8958(95 \% \mathrm{Cl} 0.8168$ - 0.9489) and 0.8542 (95\% Cl 0.7674 - 0.9179) respectively.

\section{Conclusion}

With a medically informed feature selection we were able to identify old anterior MI with $100 \%$ sensitivity and specificity by all three models in this study, and old inferior MI with $100 \%$ sensitivity and specificity by Random Forest Model. If the data set can be improved it is possible to utilize these machine learning models in hospital setting to identify cardiac emergencies by incorporating them into cardiac monitors, until trained personnel become available.

\section{Background}

Since its development in 1902, Electrocardiogram (ECG) has evolved steadily to the cost effective, simple and non-invasive 12 lead ECG used in the modern age, which is one of the most widely used investigations worldwide (1). It remains a cornerstone investigation in the medical field, utilized for the diagnosis of countless medical conditions including cardiac conditions such as acute coronary syndromes and arrhythmias and non- cardiac conditions such as electrolyte imbalances. A standard 12 lead ECG records information from 6 limb leads namely lead I, II, III, aVR, aVL and aVF and 6 chest leads from V1-V6. These leads look at the heart from different directions and record the electrical signals accordingly and the standard ECG has a $p$ wave, QRS complex and T wave (1).

The interpretation of ECG is based on the understanding of normal electrical patterns produced by the electrical activity of the heart and the variations in specific conditions. This pattern recognition process requires targeted knowledge and depends on the ability and training of the interpreter. With the development of machine learning techniques, it is possible to train a model to recognize these patterns and interpret the ECG tracing.

There have been models reported which aimed to detect arrhythmias by classifying ECGs as normal and abnormal and to narrow down the specific arrhythmia by classifying them into multiple classes (2,3). However, as a review done in 2017 reveals, studies regarding myocardial infarction (MI) and the affected territory are few (4). A vast amount of information can be gathered using the ECG, from the presence of an MI up to the affected area, timing and complications. The ECG changes of an MI depend on the lead and the timing and may include tall T waves in the initial stage, ST segment elevation, left bundle branch block, prolonged QRS 
complex, increased heart rate (reduced R-R interval) and q waves in an old Ml. According to the affected territory, these changes may be seen in different leads. For example, in leads II, III and aVF in an inferior MI and in the chest leads in an anterior MI (5).

Therefore, with these changes in mind we aimed to create a machine learning model utilizing existing ECG data to detect an MI along with the information regarding the affected territory and timing.

\section{Materials And Methods}

The dataset used was obtained from University of California, Irvine (UCI) Machine Learning Repository, which contained 279 predictor variables of 452 observations (6). The variables included age, sex, height, weight and attributes derived from standard 12 lead ECG tracings. Each observation had been assigned one of sixteen possible outcomes.

The original dataset was filtered to obtain observations categorized as Normal (class 1), Ischemic changes (class 2), Old Anterior MI (class 3) and Old Inferior Ml (class 4).

The dataset was randomly split into a training set (70\%) and a test set (30\%). Ninety nine features were selected based on the ECG changes that are observed following a Ml. They are heart rate, the amplitudes of $\mathrm{q}, \mathrm{R}$ and $\mathrm{T}$ waves and the durations of the QRS complexes along with the age and the sex of the subject (Table 1).

Table 1 Selection of ECG features based on medical knowledge

\begin{tabular}{|llll|}
\hline Feature & & Number of leads & Number of predictors \\
\hline Age & & 1 \\
\hline Sex & & 1 \\
\hline Heart rate & & 1 \\
\hline \multirow{3}{*}{ Amplitudes } & Q wave amplitude & 12 & \\
\cline { 2 - 4 } & R wave amplitude & 12 & 12 \\
\cline { 2 - 4 } & T wave amplitude & 12 & 12 \\
\hline QRS Duration & Q wave duration & 12 & 12 \\
\cline { 2 - 4 } & R wave duration & 12 & 12 \\
\cline { 2 - 4 } & S wave duration & 12 & 12 \\
\cline { 2 - 4 } & R' wave duration & 12 & 12 \\
\cline { 2 - 4 } & S' wave duration & 12 & 12 \\
\hline Total & & & 99 \\
\hline
\end{tabular}

26 predictors ( $\mathrm{R}^{\prime}$ and $\mathrm{S}^{\prime}$ waves of all the 12 leads and Q wave amplitudes of V2 and V3 leads) that had near-zero variance across the observations were excluded. The selected 73 features were scaled to have a mean of 0 and standard deviation of 1 .

Three machine learning classification models (Bootstrap Aggregation Decision Trees, Random Forest, Artificial Neural Network) were trained using the training dataset, following data up-sampling to overcome class imbalances, optimizing for the Kappa statistic, which is a measure of inter-rater reliability which takes the possibility of percentage agreement occurring by chance, into account.

Parameter tuning of the models was done with 10 fold repeated cross validation. Cross validation folds were sampled identically to make inter-model performance evaluation possible using accuracy and Kappa statistics. 
Performance of the final model was evaluated using sensitivity, specificity, positive predictive value, negative predictive value and balanced accuracy for each class for the test dataset.

The evaluation metrices are summarized below.

Accuracy $=$ Number of correct predictions $/$ The total number of predictions

Sensitivity $=$ True positive $/($ True positive + False negative $)$

Specificity $=$ True negative $/($ True negative + False positive $)$

Positive predictive value $=$ True positive $/($ True Positive + False positive $)$

Negative predictive value $=$ True negative $/($ True negative + False negative $)$

Sensitivity=TruepositivesTruepositives+Falsenegatives

Specificity=TruenegativesTruenegatives + Falsepositives

\section{Results}

The Bootstrap Aggregation classifier had a mean accuracy of 0.9788 and a mean Kappa value of 0.9718 for the 100 cross validation resamples.

The Random Forests model yielded the highest cross validation accuracy (0.9927) and Kappa metric (0.9902) out of the three models, with 3 randomly selected variables for splitting each tree node (Figure 1).

The Feed forward Artificial Neural Network was a Multi-layer Perceptron with one hidden layer (Figure 2). This model yielded the highest cross validation accuracy (0.9772) with 10 hidden units (Figure 3 ).

The distributions of the evaluation matrices of the cross validation samples and the scatter plot matrix for Kappa statistic of the three models are depicted in following figures (Figure 4, 5, 6).

All the three models had excellent cross validation accuracies.

The previously unseen test data split was used to evaluate the final model performances.

The Random Forests model predicted the classes of the test dataset with an overall accuracy of $0.9167(95 \% \mathrm{Cl} 0.8424-0.9633)$ with $100 \%$ sensitivity and specificity for identifying old anterior and old inferior myocardial infarctions.

The overall accuracies of the Bootstrap Aggregation Decision Trees and the Multi-layer Perceptron models were $0.8958(95 \% \mathrm{Cl}$ $0.8168-0.9489)$ and $0.8542(95 \% \mathrm{Cl} 0.7674-0.9179)$. Both these models could identify old anterior myocardial infarctions with $100 \%$ sensitivity and specificity.

The confusion matrices and evaluation matrices of the three models are summarized below (Tables 2-7).

\section{Random Forests model}

Table 2 Confusion matrix of Random Forest Model 


\begin{tabular}{|lllll|}
\hline \multirow{2}{*}{ Original label of the class } & \multicolumn{5}{c|}{ Radom forest Model identified as } \\
\cline { 2 - 5 } & Class 1 & Class 2 & Class 3 & Class 4 \\
\hline Class 1 & 71 & 6 & 0 & 0 \\
\hline Class 2 & 2 & 10 & 0 & 0 \\
\hline Class 3 & 0 & 0 & 5 & 0 \\
\hline Class 4 & 0 & 0 & 0 & 2 \\
\hline
\end{tabular}

Table 3 Performance of the Random Forest model on the test data

\begin{tabular}{|llllll|}
\hline & Sensitivity & Specificity & Positive Predictive Value & Negative Predictive Value & Balanced Accuracy \\
\hline Class 1 & 0.9726027 & 0.7391304 & 0.9220779 & 0.8947368 & 0.8558666 \\
\hline Class 2 & 0.6250000 & 0.9750000 & 0.8333333 & 0.9285714 & 0.8000000 \\
\hline Class 3 & 1.0000000 & 1.0000000 & 1.0000000 & 1.0000000 & 1.0000000 \\
\hline Class 4 & 1.0000000 & 1.0000000 & 1.0000000 & 1.0000000 & 1.0000000 \\
\hline
\end{tabular}

\section{Bootstrap Aggregation Decision Trees Model}

Table 4 Confusion Matrix of Bootstrap Aggregation Decision Trees Model

\begin{tabular}{|lllll|}
\hline & Class 1 & Class 2 & Class 3 & Class 4 \\
\hline Class 1 & 69 & 7 & 0 & 1 \\
\hline Class 2 & 1 & 10 & 0 & 1 \\
\hline Class 3 & 0 & 0 & 5 & 0 \\
\hline Class 4 & 0 & 0 & 0 & 2 \\
\hline
\end{tabular}

Table 5 Performance of the Bootstrap Aggregation Decision Trees Model on the test data

\begin{tabular}{|llllll|}
\hline & Sensitivity & Specificity & Positive Predictive Value & Negative Predictive Value & Balanced Accuracy \\
\hline Class 1 & 0.9857143 & 0.6923077 & 0.8961039 & 0.9473684 & 0.8390110 \\
\hline Class 2 & 0.5882353 & 0.9746835 & 0.8333333 & 0.9166667 & 0.7814594 \\
\hline Class 3 & 1.0000000 & 1.0000000 & 1.0000000 & 1.0000000 & 1.0000000 \\
Class 4 & 0.5000000 & 1.0000000 & 1.0000000 & 0.9787234 & 0.7500000 \\
\hline
\end{tabular}

\section{Multi-layer Perceptron Model}

Table 6 Confusion Matrix of Multi-layer Perceptron Model 


\begin{tabular}{|lllll|}
\hline & Class 1 & Class 2 & Class 3 & Class 4 \\
\hline Class 1 & 65 & 8 & 0 & 4 \\
\hline Class 2 & 2 & 10 & 0 & 0 \\
\hline Class 3 & 0 & 0 & 5 & 0 \\
\hline Class 4 & 0 & 0 & 0 & 2 \\
\hline
\end{tabular}

Table 7 Performance of Multi-layer Perceptron Model on the test data

\begin{tabular}{|llllll|}
\hline & Sensitivity & Specificity & Positive Predictive Value & Negative Predictive Value & Balanced Accuracy \\
\hline Class 1 & 0.9701493 & 0.5862069 & 0.8441558 & 0.8947368 & 0.7781781 \\
\hline Class 2 & 0.5555556 & 0.9743590 & 0.8333333 & 0.9047619 & 0.7649573 \\
\hline Class 3 & 1.0000000 & 1.0000000 & 1.0000000 & 1.0000000 & 1.0000000 \\
\hline Class 4 & 0.3333333 & 1.0000000 & 1.0000000 & 0.9574468 & 0.6666667 \\
\hline
\end{tabular}

\section{Discussion}

The UCI machine learning dataset has been utilized by previous studies for the detection of heart disease, mainly arrhythmias. A modular neural network model proposed by Jadhav et al., aimed to detect arrhythmias by classifying the ECG tracings as 'normal' and 'abnormal'. It was found that the model classified the tracings into the two classes with $82.22 \%$ accuracy (2). Batra et al., also utilized multiple machine learning algorithms for the classification of arrhythmias into multiple classes. The maximum accuracy achieved was $84.82 \%$ using Support Vector Machine (SVM) algorithm and gradient boosting, in conjunction (3). A model proposed by Mitra et al., utilized neural networks for the classification of arrhythmias into 'normal' and 'arrhythmia'. It was found that an accuracy of $87.71 \%$ was achievable using this method (7).

The feature extraction and reduction was carried out by correlation-based feature subset selection. A notable characteristic in the above models is that the feature selection was not performed based on medical knowledge regarding the diseases but rather by mathematical feature reduction techniques. It is possible that this may be responsible for the relatively similar accuracies which do not exceed $90 \%$. Furthermore, this data set has not been utilized specifically for the detection of myocardial infarctions and its subsets.

It is evident by this study that the detection and classification of myocardial infarction with regard to timing and territory can be achieved with a relatively higher accuracy. The better model performances demonstrated in our study may be attributed to the feature selection technique utilized, which was predominantly based on the known electrophysiological changes occurring during a myocardial Infarction. All three models were able to identify old anterior MI with $100 \%$ sensitivity and $100 \%$ specificity, with the random forest model being able to identify old inferior MI with similar matrices as well. Therefore, this study highlights the potential real world applications of this technology in the healthcare sector. The availability of an interpretation along with the tracing would be of paramount importance in conditions like myocardial infarctions where time is of the essence. The need to wait for trained personnel for the interpretation of ECG can be avoided and hence delays minimized. Patients requiring emergent care can be identified in settings with a large patient load. In addition, implanted cardioverter defibrillators (ICDs) and pacemakers of patients can also be equipped with this technique, enabling the detection of $\mathrm{Ml}$ as well. Hence, it is evident that further studies to explore the boundaries of these new upcoming leaps in technology should be performed for the improvement of medical diagnostics.

\section{Conclusion}

We used three machine learning models to detect $\mathrm{Ml}$ along with timing and territory, using data derived from ECG tracings. The overall accuracies obtained by Random Forests, Bootstrap Aggregation Decision Trees and Multi-layer Perceptron were 0.9167, 0.8958 and 0.8542 respectively. Furthermore, all the models could identify old anterior Mls with a specificity and sensitivity of $100 \%$.

Page 6/12 
This study can be expanded by using a more extensive data set, to include more detail regarding timing and territories, as well as the detection and classification of arrhythmias. Hence, it can be concluded that these models can be utilized in hospital settings to minimize the delays until trained personnel are available for ECG interpretation and to alert the staff of a potential emergency.

\section{List Of Abbreviations}

ECG - Electrocardiogram

MI - Myocardial Infarction

ICD - Implanted Cardioverter Defibrillator

\section{Declarations}

\section{Ethics approval and consent to participate}

Not applicable

\section{Consent for publication}

Not applicable

\section{Availability of data and materials}

The dataset analyzed during the current study are available in the University of California, Irvine (UCI) Machine Learning Repository , [ http://archive.ics.uci.edu/ml//datasets/Arrhythmia ]

\section{Competing interests}

The authors declare that they have no competing interests

\section{Funding}

Study was self-funded

\section{Authors' contributions}

MDS extracted the data and designed the models while IA analyzed the data and drafted the manuscript. ESK also contributed to drafting the manuscript and S was in charge of overall supervision, interpretation and revising of the article.

All authors have approved the submitted version and to have agreed both to be personally accountable for the author's own contributions and to ensure that questions related to the accuracy or integrity of any part of the work, even ones in which the author was not personally involved, are appropriately investigated, resolved, and the resolution documented in the literature.

\section{Acknowledgements}

Not applicable

\section{References}

1. Electrocardiography: Overview, ECG Indications and Contraindications, Preparation [Internet]. Emedicine.medscape.com. 2020 [cited 28 September 2020]. Available from: https://emedicine.medscape.com/article/1894014-overview

2. Jadhav S, Nalbalwar S, Ghatol A. ECG arrhythmia classification using modular neural network model. 2010 IEEE EMBS Conference on Biomedical Engineering and Sciences (IECBES). 2010;

3. Batra A. Classification of Arrhythmia Using Conjunction of Machine Learning Algorithms and ECG Diagnostic Criteria. [Internet]. 2016 [cited 28 September 2020];. Available from: https://www.semanticscholar.org/paper/Classification-of-Arrhythmia-Using- 
Conjunction-of-Batra/96c4234eb0e9d3443d09bca7d21caeef8733b26b

4. Roopa C, Harish B. A Survey on various Machine Learning Approaches for ECG Analysis [Internet]. 2017 [cited 28 September 2020]. Available from:

https://www.researchgate.net/publication/317400128_A_Survey_on_various_Machine_Learning_Approaches_for_ECG_Analysis

5. Burns E. Myocardial Ischaemia • LITFL • ECG Library Diagnosis [Internet]. Life in the Fast Lane • LITFL • Medical Blog. 2020 [cited 28 September 2020]. Available from: https://litfl.com/myocardial-ischaemia-ecg-library/

6. Altay Guvenir, BurakAcar, GulsenDemiroz, AyhanCekin "A Supervised Machine Learning Algorithm for Arrhythmia Analysis." Proceedings of the Computers in Cardiology Conference, Lund, Sweden, 1997.

7. Mitra M, Samanta R. Cardiac Arrhythmia Classification Using Neural Networks with Selected Features. Procedia Technology. 2013;10:76-84.

8. McHugh ML. Interrater reliability: the kappa statistic. Biochem Med (Zagreb). 2012;22(3):276-282.

\section{Figures}

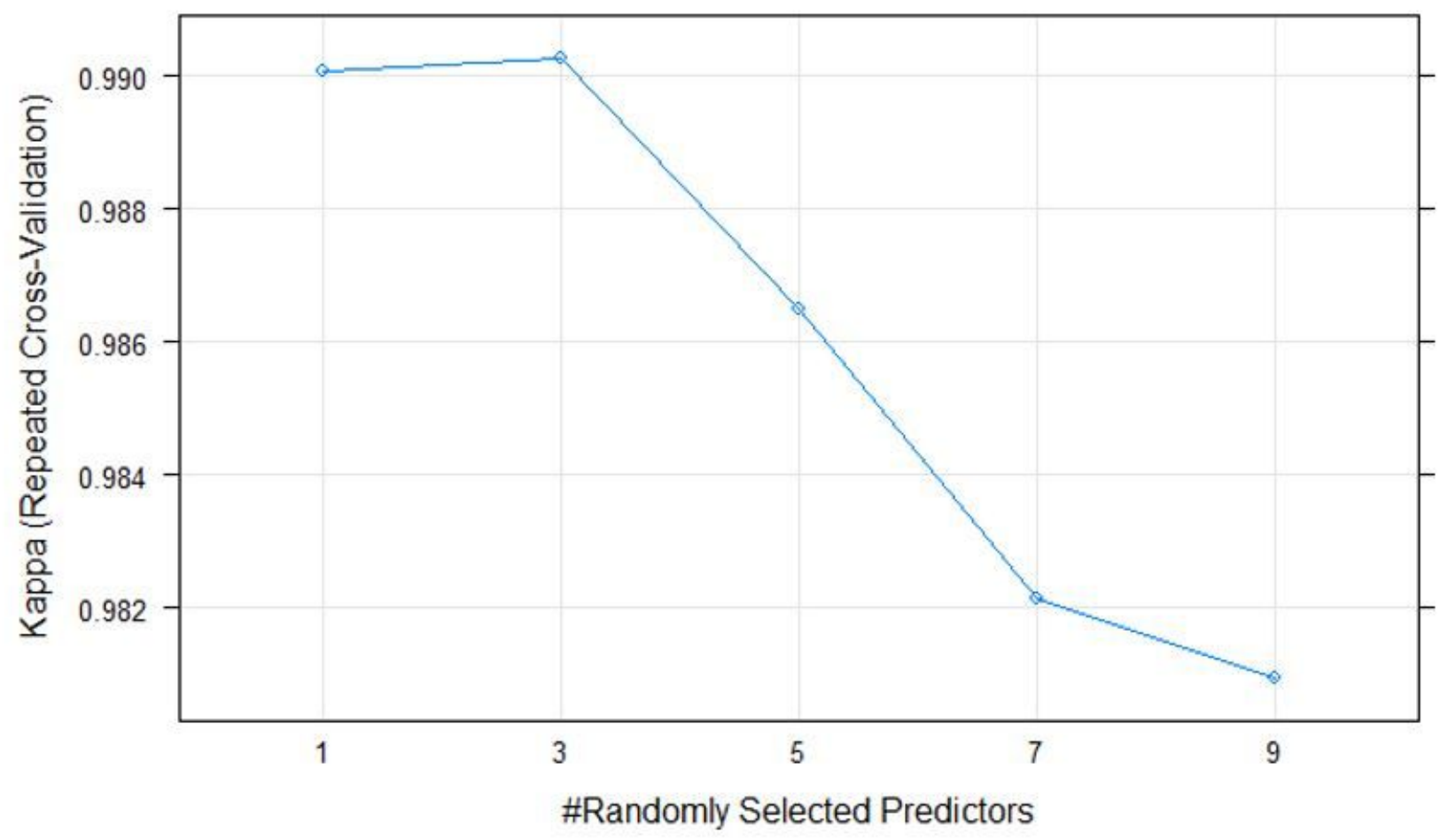

Figure 1

Cross validation Kappa statistics for different numbers of randomly selected predictors of the Random Forests model. 


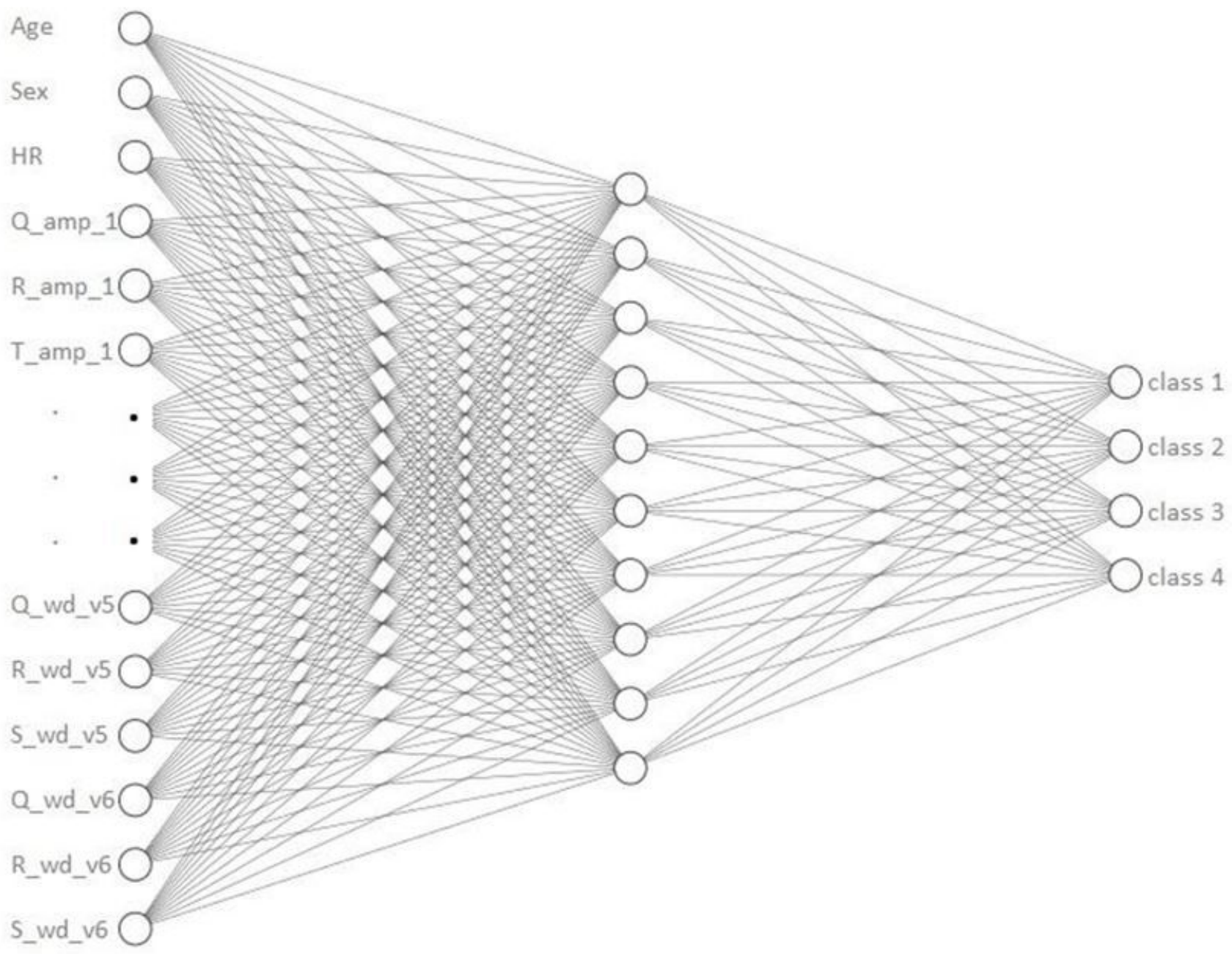

Input Layer $\in R^{73}$

Hidden Layer $\in \mathrm{R}^{10}$

Output Layer $\in \mathbf{R}^{4}$

\section{Figure 2}

The architecture of the Perceptron 


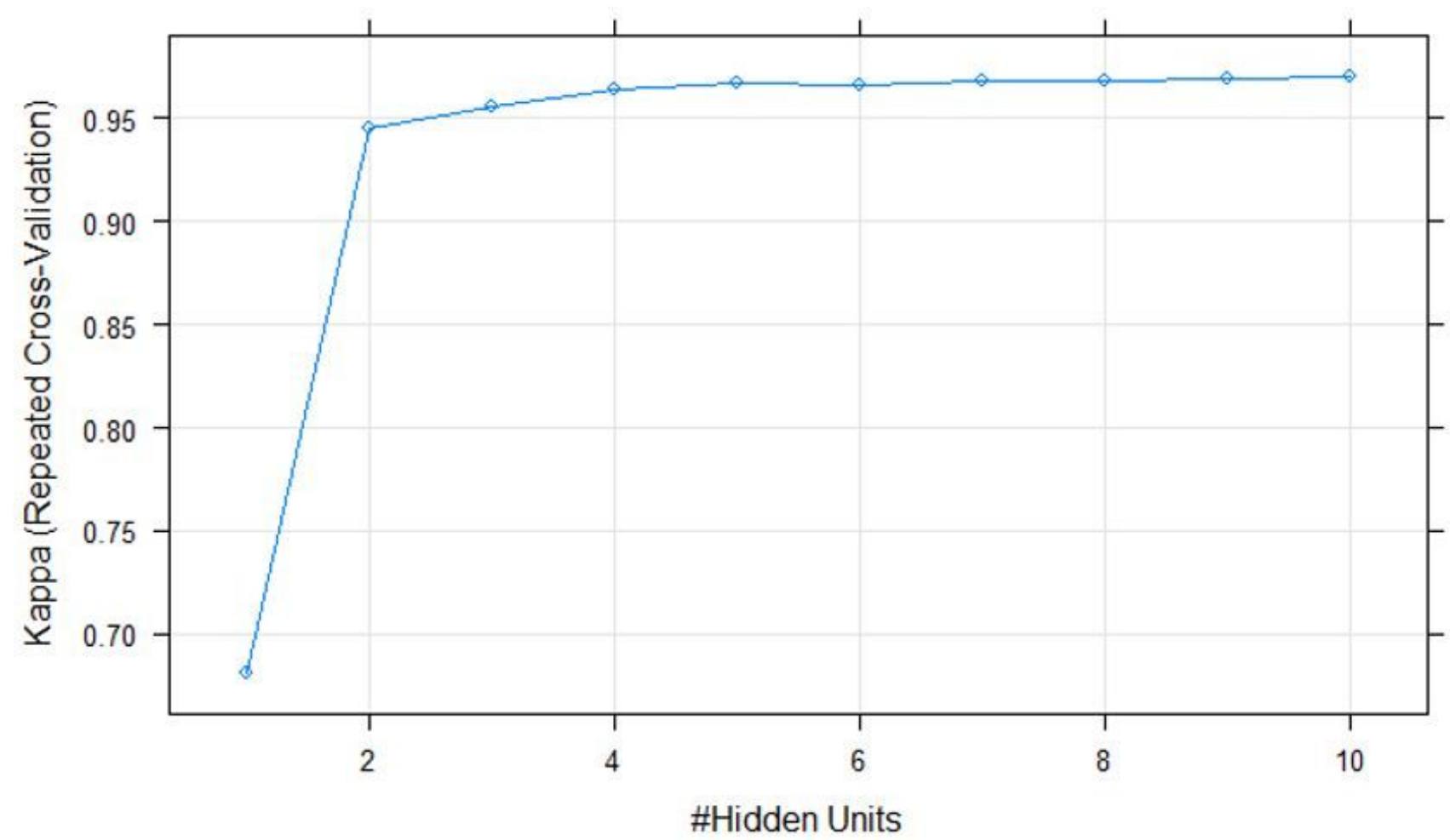

Figure 3

Cross validation Kappa statistics for different numbers hidden units of the neural network.

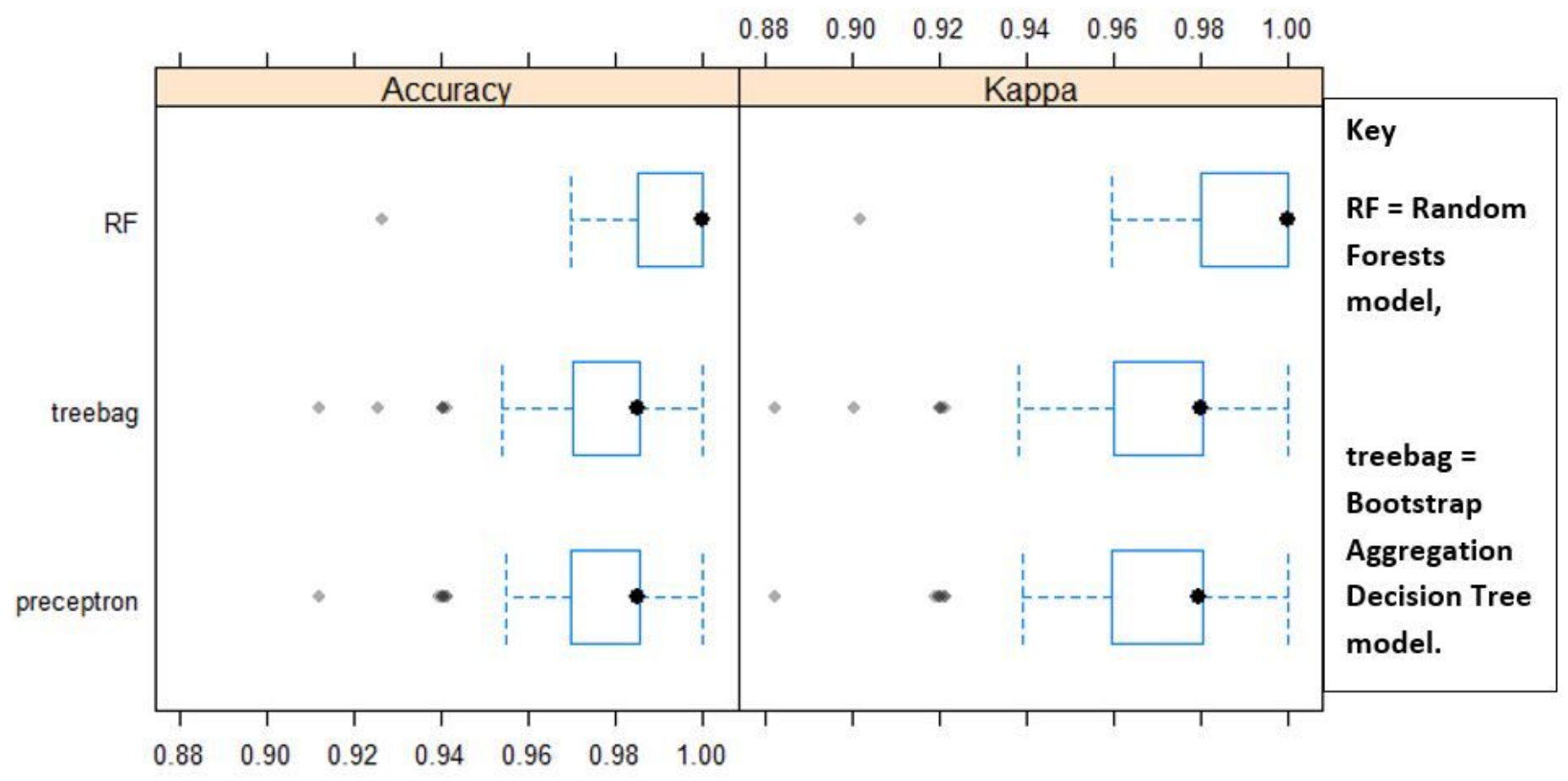

Figure 4 
Box-and-whisker plots of the resampling distributions of the accuracy and the Kappa between the models.

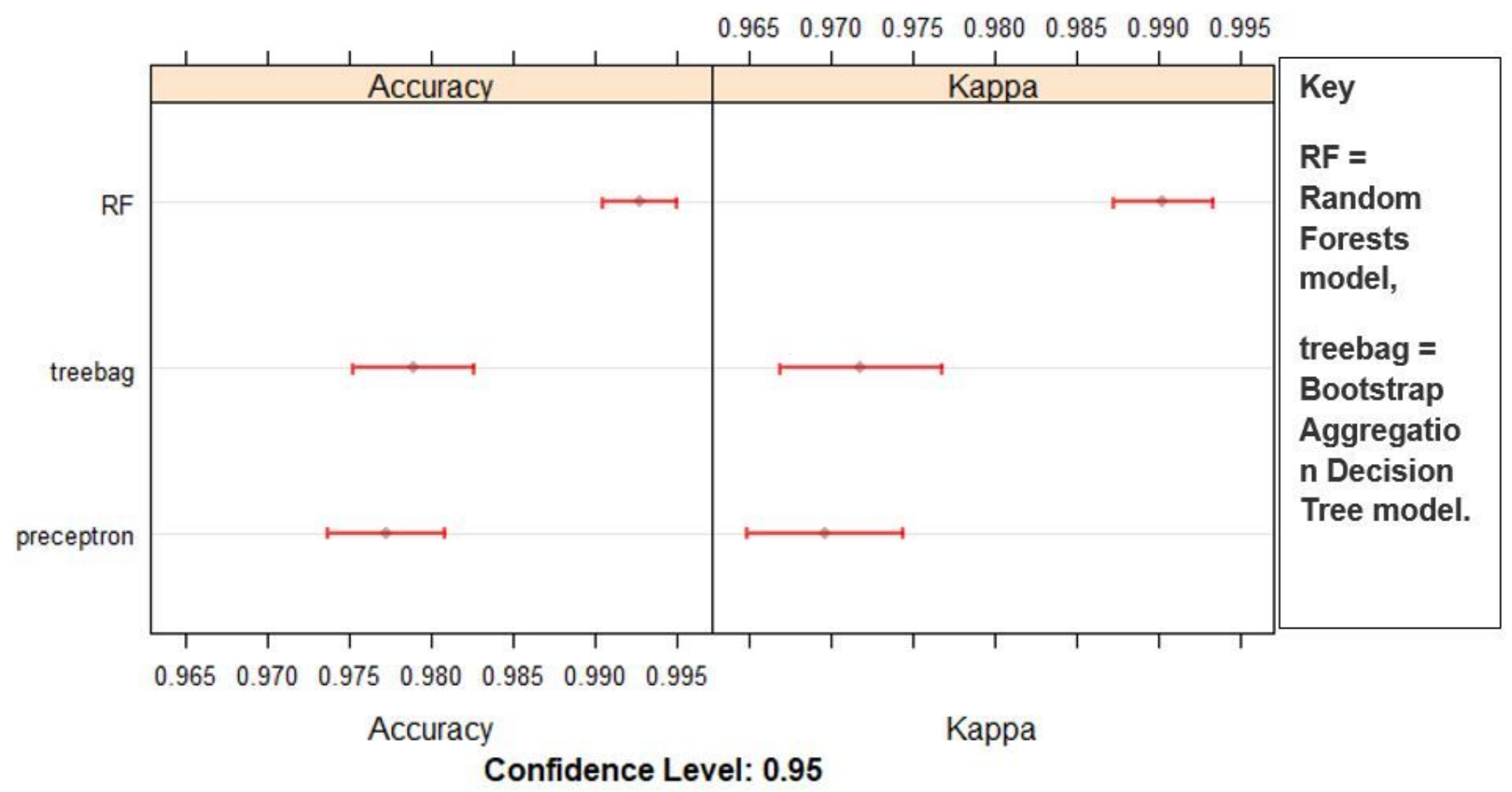

Figure 5

Resampling distributions of the accuracy and the Kappa between the models. 


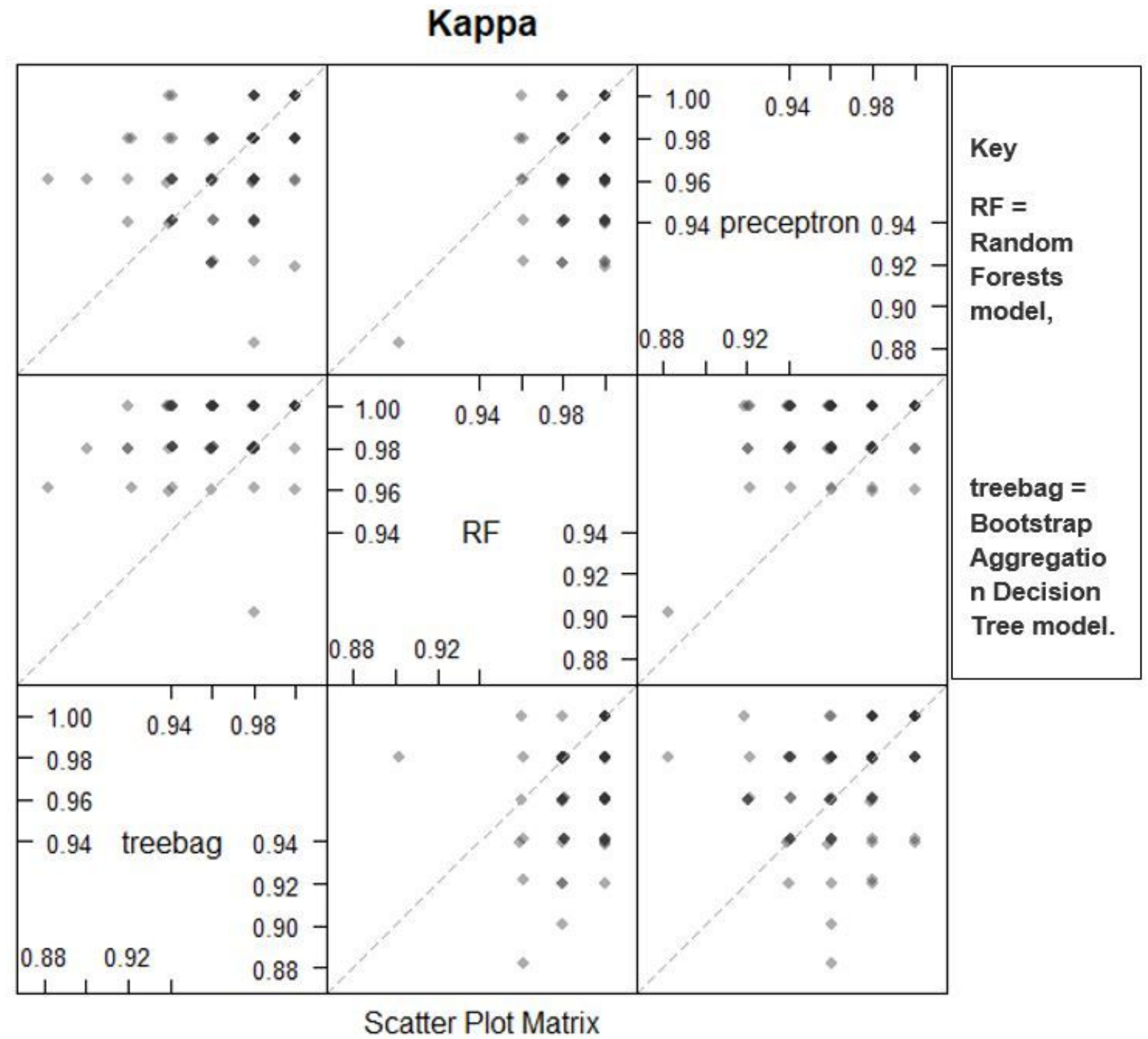

Figure 6

Scatter plot matrix of the resampling distributions of the Kappa between the models. 